\title{
Hyperbaric oxygen therapy fails to reduce hydrocephalus formation following subarachnoid hemorrhage in rats
}

\author{
Qin $\mathrm{Hu}^{1}$, Alexander Vakhmjanin ${ }^{1}, \mathrm{Bo} \mathrm{Li}{ }^{1}$, Jiping Tang ${ }^{1}$ and John $\mathrm{H}$ Zhang ${ }^{1,2^{*}}$
}

\begin{abstract}
Background \& purpose: Approximately $40 \%$ of hemorrhagic stroke survivors develop hydrocephalus. Hyperbaric oxygen (HBO) has been shown to be anti-inflammation following experimental stroke; however, its effect upon post-hemorrhagic hydrocephalus formation is not known. The objective of this study is to investigate whether HBO therapy can effectively reduce hydrocephalus formation and improve neurobehavioral functions in a rat model of subarachnoid hemorrhage (SAH).
\end{abstract}

Method: Thirty-eight male Sprague-Dawley rats (300-320 g) rats survived for 21 days from SAH by endovascular perforation or sham surgery were used. At 24 hours after SAH, HBO (3 atmospheres absolute) or normobaric oxygen (NBO) administrated for 1 hour once daily for a total of 7 days. Wire hanging and rotarod testing were conducted at 14 days after $\mathrm{SAH}$, and cognitive functions were evaluated via the Morris water maze, between day 17 to day 21 after surgery. At day 21, rats were sacrificed and cerebroventricular volumes were measured histologically.

Results: Hydrocephalus exacerbated neurological deficits after SAH, and HBO multiple treatment tendentially improved the neurobehavioral functions. Spatial learning and memory deficits were noticed after SAH, and rats with hydrocephalus showed worse learning and memory abilities and HBO treatment showed a minor improvement. In the SAH group (room air) 4 rats showed an increased ventricular volume at day 21 after SAH-induction $(n=10)$. HBO or NBO therapy did not alter the occurrence of hydrocephalus after SAH, as 4 rats in each of these groups showed an increased ventricular volume ( $n=10$ per group).

Conclusion: Multiple HBO therapy does not ameliorate hydrocephalus formation in a rat model of SAH; however, $\mathrm{HBO}$ tendentially improved the neurological functions and spatial learning and memory abilities in rats with hydrocephalus.

Keywords: Hyperbaric oxygen, Hydrocephalus, Neurobehavioral, Subarachnoid hemorrhage

\section{Introduction}

Hydrocephalus defines an expansion of the cerebroventricular system, which is associated with a decrease in brain tissue as well as compromised neurological functions. The neurological deficits resulted from hydrocephalus range from subtle neurocognitive and gait problems to severe disability requiring permanent cerebrospinal

\footnotetext{
* Correspondence: johnzhang3910@yahoo.com

'Department of Physiology and Pharmacology, Loma Linda University School of Medicine, 11041 Campus Street, Risley Hall, Room 219, Loma Linda CA 92354, USA

${ }^{2}$ Department of Neurosurgery, Loma Linda University School of Medicine, 11041 Campus Street, Risley Hall, Room 219, Loma Linda, CA 92354, USA
}

fluid (CSF) diversion. Approximately 40\% of hemorrhagic stroke survivors develop a hydrocephalus [1]. Hydrocephalus can be classified into communicating, noncommunicating or obstructive types [2]. Decreased absorption of CSF at the arachnoid granulations is defined as communicating hydrocephalus and an anatomic obstruction, as noncommunicating. Subarachnoid hemorrhage $(\mathrm{SAH})$ is a common cause of chronic communicating hydrocephalus in clinic, which affects up to $20 \%$ of the survivors [3].

Although the cause of chronic hydrocephalus after $\mathrm{SAH}$ is still uncertain, previous studies suggested that proliferation of arachnoid cells and leptomeningeal 
fibrosis, triggered by an inflammatory reaction or blood clotting products, may impair CSF flow through the arachnoid villi, resulting in hydrocephalus $[4,5]$. Current therapies for hydrocephalus rely on invasive procedures that are associated with high failure and complication rates [6]. And so far no medical treatment is effective in long-term treatment of chronic hydrocephalus. $\mathrm{HBO}$ has been shown to reduce inflammation in many chronic diseases $[7,8]$ and proved to be neuroprotective and anti-inflammation following experimental stroke [9]; however, its effect upon post-hemorrhagic hydrocephalus formation is not known. The objective of this current study is to investigate whether multiple HBO therapy can effectively reduce hydrocephalus formation and improve the neurological functions in a rat model of SAH.

\section{Materials and methods}

This study was carried out in strict accordance with the recommendations in the Guide for the Care and Use of Laboratory Animals of the National Institutes of Health. All protocols were approved by the Institutional Animal Care and Use Committee of Loma Linda University.

\section{Experiment design}

This is a retrospective study. A total of 38 adult male Sprague-Dawley rats (300-320 g, Harlan, Indianapolis, IN) survived for 21 days from $\mathrm{SAH}$ by endovascular perforation $(n=45)$ or sham surgery $(n=8)$ were used for the study. 24 hours after SAH, rats were randomly divided into 3 groups: SAH, SAH with $\mathrm{HBO}$ treatment $(\mathrm{SAH}+\mathrm{HBO}), \mathrm{SAH}$ with normobaric oxygen (NBO) treatment $(\mathrm{SAH}+\mathrm{NBO})$. Foot-fault, wire hanging and rotarod tests were conducted at post-operative day 14, and cognitive functions were evaluated via the Morris water maze, between day 17 to day 21 after surgery. At day 21, rats were sacrificed after measuring the body weight and cerebroventricular volumes were measured histologically. In $\mathrm{SAH}, \mathrm{SAH}+\mathrm{HBO}$ and $\mathrm{SAH}+\mathrm{NBO}$ groups, animals were divided into $\mathrm{SAH}$ without hydrocephalus (SAH w/t HyC) and $\mathrm{SAH}$ with hydrocephalus $(\mathrm{SAH} / \mathrm{HyC})$ respectively according to the results of cerebroventricular volume. Data were analyzed in Sham, SAH w/t HyC, SAH/HyC, SAH/HyC + HBO, SAH/HyC + NBO groups.

\section{Experimental model of $\mathrm{SAH}$ and $\mathrm{HBO}$ treatment}

The endovascular perforation model of $\mathrm{SAH}$ was performed as previously described [10]. Briefly, rats were anesthetized with $3 \%$ isoflurane in $70 \% / 30 \%$ medical-air/ oxygen, intubated and kept on artificial ventilation during surgery. Body temperature was monitored by a rectal probe and normothermia was maintained by a heating lamp. A sharpened 4-0 nylon suture was introduced into the right internal carotid artery (ICA) until resistance was felt (approximately $18 \mathrm{~mm}$ from the common carotid bifurcation). The suture was then advanced until the resistance was overcome to perforate the bifurcation of the anterior and middle cerebral arteries. The suture was withdrawn immediately after perforation. In Sham operated animals the suture was inserted into the right ICA, however no vessel perforation was performed. After suture removal the incision was closed, and rats were individually housed in heated cages until recovery.

24 hours after SAH, rats were pressurized in a research hyperbaric chamber (1300B; Sechrist) at 3 atmospheres absolutes (ATA) with $100 \%$ oxygen (flow of $22 \mathrm{~L} / \mathrm{min}$ ) for $1 \mathrm{~h}$ once daily for consecutive 7 days. Doses of $\mathrm{HBO}$ were selected based on previous studies [11]. SAH rats treated with NBO were used as a control group.

\section{Neurological scores}

At $24 \mathrm{~h}$ after SAH, Garcia test was performed by a blinded investigator as previously described with modifications [12]. The scores given to each rat at the completion of the evaluation was the summation of all 6 individual test scores (spontaneous activity, symmetry in the movement of four limbs, forepaw outstretching, climbing, body proprioception, and response to vibrissae touch). The minimum neurological score (most severe deficit) was 3 , and the maximum was 18 .

\section{Foot-fault test, wire hanging and rotarod}

At day 14 (6 days after the last HBO treatment), footfault test, wire hanging and rotarod were measured by an investigator who was blinded to the experimental groups as previously described [13]. In the foot fault test, the rats were placed on a horizontal grid floor for 2 minutes. The foot fault was defined as when the rat inaccurately placed a fore- or hindlimb and it fell through one of the openings in the grid. The number of foot-faults was recorded and was used for the statistical analysis.

The wire hanging test procedure was basically the same as that described by Shabani et al. [14] and is used to assess the muscle strength and balance. Each rat was suspended with both forepaws on a horizontal steel wire $80 \mathrm{~cm}$ long, diameter $7 \mathrm{~mm}$. The animal was held in a vertical position when its front paws were placed in contact with the wire. When the rat grasped the wire, it was released, and the latency to fall was recorded with a stopwatch. Rats were randomly tested and each animal was given three trials with a $30 \mathrm{~min}$ inter-trial rest interval.

Rotarod was performed at day 14 after SAH to test coordination and balance as previously described [15]. The rotarod consists of a rotating horizontal cylinder (7 $\mathrm{cm}$ diameter) that is divided into 9.5 - $\mathrm{cm}$-wide lanes. When placed into a lane, an animal must continuously 
walk forward to avoid falling off the cylinder. Latency to fall off was detected and recorded by a photobeam circuit. Two consecutive trials were administered, in which the cylinder started turning at $5 \mathrm{rpm}$, and was accelerated by $2 \mathrm{rpm}$ every $5 \mathrm{sec}$. To control for any potential learning effect due to previous rotarod exposure, an additional set of more difficult trials was added in which the cylinder started turning at $10 \mathrm{rpm}$, and was accelerated by 2 rpm every 5 sec.

\section{Morris water maze}

At day 17 to day 21 after SAH, Morris water maze was performed in a blinded setup as previously described [15]. In brief, it consists of three trials (cued, spatial, and probe) done over four consecutive days. All trials lasted a maximum of $60 \mathrm{sec}$. The cued trials have a visible platform above the water level where the animals were allowed to remain on the platform for $10 \mathrm{sec}$ after finding it or being guided to it. The spatial trials have the platform submerged in the water. Once released, they were allowed to swim in search of the platform. Here the time taken to find the platform measured spatial learning. The probe trial; platform is removed completely and rats were allowed to swim again in search of the platform measuring spatial memory.

\section{Nissl staining and brain ventricular volume}

Rats were fatally anesthetized with isoflurane $(\geq 5 \%)$ followed by cardiovascular perfusion with ice-cold PBS and $10 \%$ formaldehyde. Brains were removed and postfixed/cryoprotected in 10\% formaldehyde/30\% sucrose for 3 days, embedded in mounting medium and frozen in liquid nitrogen. For all neuropathological analyses $10 \mu \mathrm{m}$ thick coronal sections were cut on a cryostat (Leica Microsystems LM3050S), mounted on poly-L-lysinecoated slides and Nissl stained. Three Nissl-stained coronal sections at bregma $0(+/-250 \mu \mathrm{m})$ were randomly chosen and photographed under light microscopy. Morphometric analysis of these slides was performed by computer-assisted (Photoshop CS4, Adobe) hand delineation of the ventricles and the total brain area. The relative ventricle area was calculated as ventricle area/total brain area, and took the average of the three slices as the value for each animal.

\section{Statistical analyses}

Data were expressed as the mean \pm SEM. The analysis of the data was performed using GraphPad Prism software. Statistical differences among groups were analyzed by using one-way analysis of variance followed by the Turkey method. A value of $p<0.05$ was taken as significant.

\section{Results}

SAH rats showed neurological deficits at $24 \mathrm{~h}$ and no difference between groups before treatment

At 24 hours after SAH, there was a very significant impairments in neurological functions compared with Sham group (Figure 1, $p<0.05$, compared with Sham). There is no significant difference between $\mathrm{SAH}$ w/t $\mathrm{HyC}, \mathrm{SAH} /$ $\mathrm{HyC}, \mathrm{SAH} / \mathrm{HyC}+\mathrm{HBO}$ or $\mathrm{SAH} / \mathrm{HyC}+\mathrm{NBO}$ groups before treatment. The results showed our SAH model is successful and stable.

Multiple HBO treatment neither altered the occurrence of hydrocephalus nor affected the ventricular volume after SAH

At 21 days after SAH, 4 rats showed an increased ventricular volume compared with Sham (Figure 2, (5.621 \pm $0.704) \%$ vs. $(1.844 \pm 0.129) \%, p<0.05$ compared with Sham); 6 rats showed no significant difference in ventricular volume (Figure $2,(2.337 \pm 0.223) \%$ vs. $(1.844 \pm 0.129) \%$, $p>0.05$ compared with Sham); the occurrence of hydrocephalus is $40 \%$. $\mathrm{HBO}$ or NBO treatment did not alter the occurrence of hydrocephalus, as 4 rats (40\%) in each of these groups. Multiple $\mathrm{HBO}$ treatment did not affect the cerebroventricular volumes in the hydrocephalic animals (Figure 2, $(5.181 \pm 0.807) \%$ vs. $(5.621 \pm 0.704) \%, p>0.05$ compared with $\mathrm{SAH} / \mathrm{HyC}$ ).

Multiple HBO treatment improved the performance of foot-fault and rotarod in hydrocephalic animals

At 14 days after SAH, animals showed significant deficits in foot fault in left forelimb than in Sham group (Figure 3A). Hydrocephalus deteriorated the performance

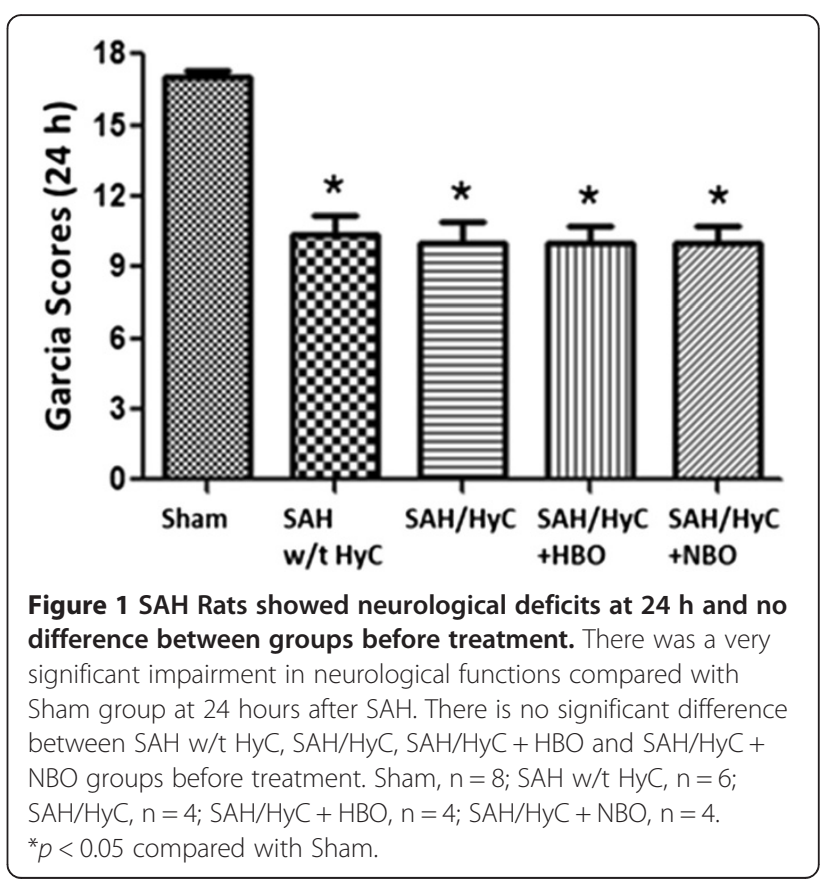




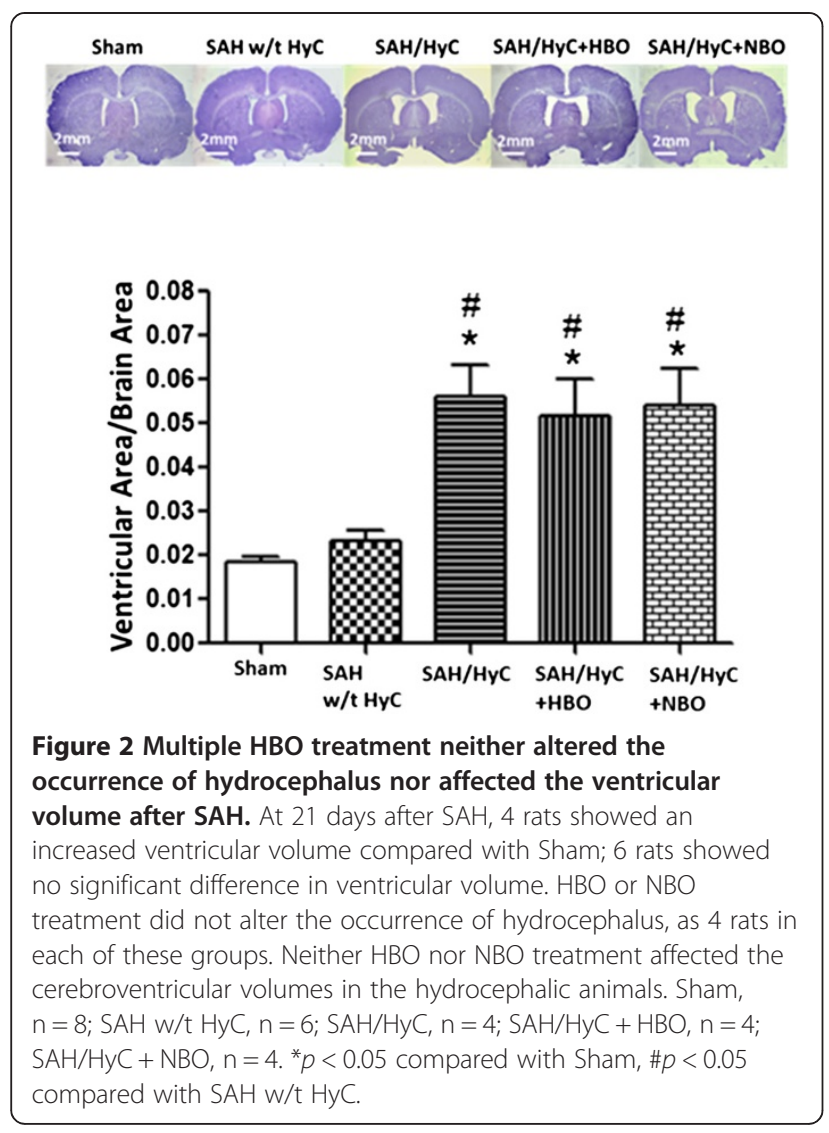

(Figure 3A, $p<0.05$ compared with SAH w/t HyC). HBO treatment did not significantly ameliorate the deficits of foot fault in hydrocephalic animals (Figure 3A, $p>0.05$, compared with $\mathrm{SAH} / \mathrm{HyC}$ ), but showed tendency to improve the performance and removed the significance between SAH w/t HyC (Figure 3A, $p>0.05$, compared with $\mathrm{SAH}$ w/t $\mathrm{HyC}$ ). After $\mathrm{SAH}$, there is no significant reduce of muscle strength in hydrocephalic animals (Figure 3B, $p>0.05$, compared with $\mathrm{SAH} w / t \mathrm{HyC}$ ), and $\mathrm{HBO}$ treatment did not increase the time of wire hanging (Figure 3B, $p>0.05$, compared with $\mathrm{SAH} w / \mathrm{t} \mathrm{HyC}$ ). In accelerating rotarod, hydrocephalic animals more easily fell off from the rotarod than non-hydrocephalic rats (Figure $3 \mathrm{C}, p<0.05$, compared with $\mathrm{SAH} w / \mathrm{t} \mathrm{HyC}$ ), and $\mathrm{HBO}$ treatment showed minor improvement by eliminating the significance between SAH w/t HyC. NBO treatment showed no improvements in the performance foot-fault, wire hanging and rotarod.

There was a significant decrease of body weight at 21 days after SAH (Figure 3D, $p<0.05$, compared with Sham). Hydrocephalus did not affect the body weight remarkably (Figure 3D, $p>0.05$, compared with SAH w/t $\mathrm{HyC}$ ), and $\mathrm{HBO}$ treatment has no effect on body weight in long-term (Figure 3D, $p>0.05$, compared with $\mathrm{SAH} /$ $\mathrm{HyC})$.

\section{Multiple HBO treatment improved spatial learning and memory abilities in hydrocephalic animals}

There were spatial learning and memory deficits at 21 days after SAH. When compared with Sham group, the animals in SAH w/t HyC group had a significantly greater distance moved from the target (Figure 4A), need more time to reach the platform (Figure 4B), showed less frequency in target quadrants (Figure 4C), spent less time in the probe quadrants (Figure 4D). Hydrocephalus exacerbated the performance (Figure 4D, $p<0.05$, compared with $\mathrm{SAH}$ w/t $\mathrm{HyC}$ ). Multiple $\mathrm{HBO}$ treatment did not showed significant improvement of memory and learning abilities when compared with $\mathrm{SAH} / \mathrm{HyC}$ group (Figure 4, $p>0.05$ compared to $\mathrm{SAH} / \mathrm{HyC}$ ), but removed the difference between SAH w/t HyC group (Figure 4, $p>0.05$ compared to $\mathrm{SAH}$ w/t $\mathrm{HyC}$ ).

\section{Discussion}

In the present study, we observed increased ventricular volume and deteriorated neurobehavioral after SAH induced by endovascular perforation in rats. The data showed that $40 \%$ of the animals developed hydrocephalus which was associated with ventricular dilation - the typical characteristics of chronic communicating hydrocephalus. The incidence of hydrocephalus is consistent with its clinical relevance and our previous study [16]. Repeated HBO treatment did not ameliorate hydrocephalus formation but tendentially improve the neurological deficits and spatial learning and memory abilities. Our data indicates multiple HBO treatment is beneficial for the recovery of neurological functions after SAH; however, there is no improvement in hydrocephalus.

The treatment of hydrocephalus can be divided into two main groups: surgical and medical treatment [17]. Despite increasing improvements in surgical techniques and instruments, hydrocephalus remains quite a challenge for neurosurgery and there is still a high complication rate. Rapid and effective interventions with medicine are suggested as promising strategies to minimize the occurrence of hydrocephalus. Inflammatory reaction caused by blood clotting products after $\mathrm{SAH}$ is claimed to be one of the perpetrators for communicating hydrocephalus $[4,18]$. HBO has been shown to reduce inflammation in diabetic foot [8], traumatic brain injury [19], and also in experimental stroke $[9,20]$. HBO treatment can suppress inflammation by reducing neutrophil-endothelial adhesion through S-nitrosation [21], reducing the toll-like receptor signaling pathway [22], increasing heme oxygenase-1 [23] and inhibiting matrix metalloproteinase-9 [24]. In this study, HBO seems invalid to prevent the development of hydrocephalus after SAH. The results suggested that HBO treatment might not work for anti-inflammation when begin at 24 hours after SAH. However, multiple HBO treatment did improve neurobehavioral deficits and spatial 

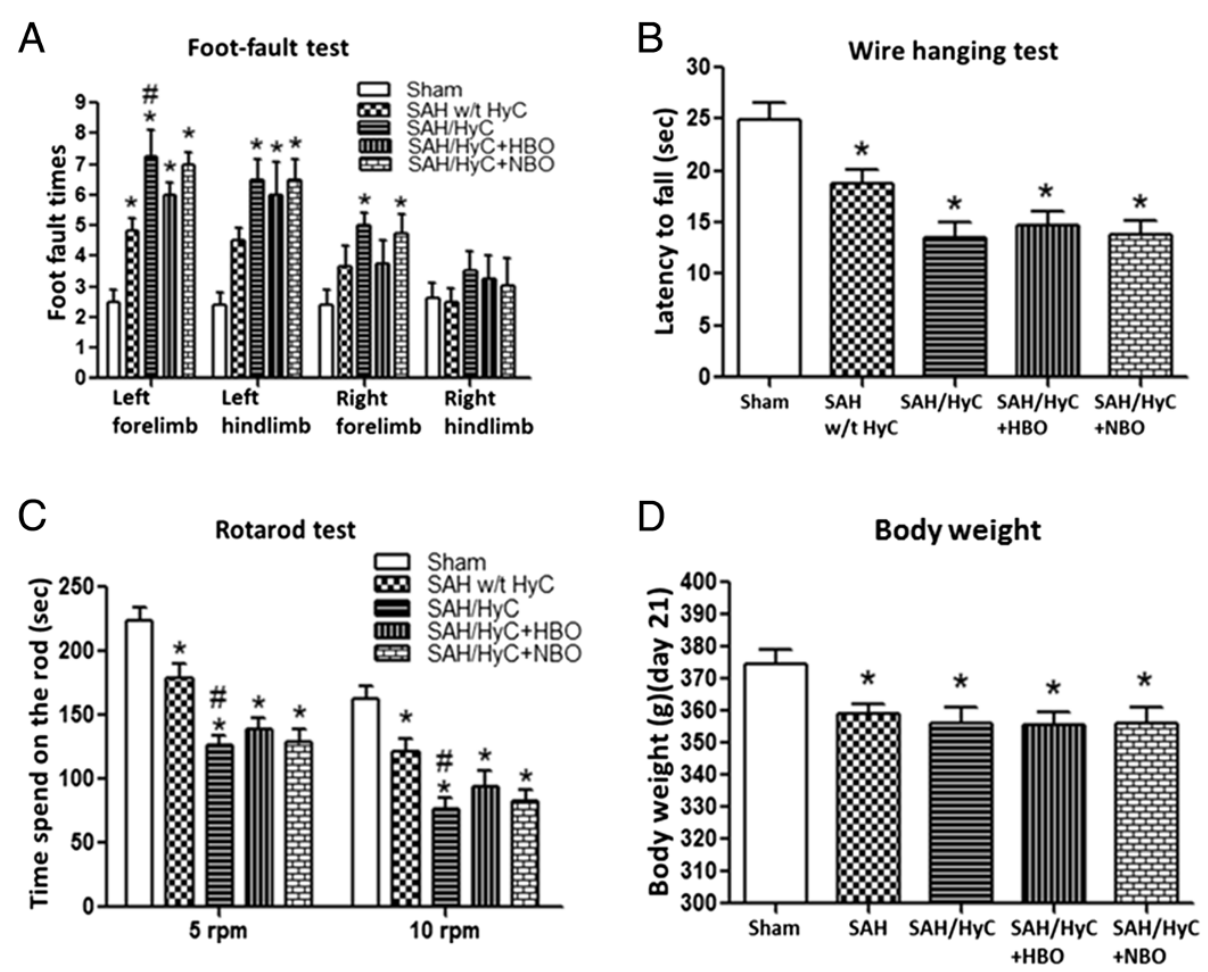

Figure 3 Multiple HBO treatment mildly improved the neurological deficits in hydrocephalic animals. (A) 14 days after SAH, the foot fault times in left forelimb were significantly higher than that in Sham group. Hydrocephalus deteriorated the performance, and HBO treatment showed tendency to improve the performance and removed the significance between SAH w/t HyC. (B) After SAH, there is no significant reduce of muscle strength between hydrocephalic and non-hydrocephalic animals. HBO treatment did not increase the time of wire hanging. (C) In accelerating rotarod, hydrocephalic animals more easily fell off from the rotarod than non-hydrocephalic rats, and HBO treatment showed minor improvement by eliminating the significance between SAH w/t HyC. (D) At 21 days after surgery, there is significant difference in body weight between Sham-operated animals and the SAH-operated animals, but no difference between the SAH-operated with $\mathrm{HBO}$ or NBO treatment groups. Sham, $n=8 ;$ SAH w/t HyC, $n=6 ; S A H / H y C, n=4 ; S A H / H y C+H B O, n=4 ; S A H / H y C+N B O, n=4 .{ }^{*} p<0.05$ compared with Sham, \#p $<0.05$ compared with SAH w/t HyC.

learning and memory abilities in long-term in hydrocephalic animals. The improvements of neurological functions and cognitive functions may result from the neuroprotection of $\mathrm{HBO}$ and neurogenesis after multiple $\mathrm{HBO}$ treatment. HBO treatment has been proved to prevent neuron apoptosis in experimental stroke [25,26]. And our previous studies have demonstrated that delayed and multiple HBO treatment can promote the recovery of neurobehavior through promoting neurogenesis in focal brain ischemic rats [11].

In our study, we observed obviously worse neurological deficits and cognitive functions in hydrocephalic animals than non-hydrocephalic animals at 21 days after $\mathrm{SAH}$. We detected the neurological functions at $24 \mathrm{~h}$ after SAH and there is no difference between the SAH operated animals, which excluded the difference in the severity of SAH. These findings support the conclusion that impairments in cognition and motor skills corresponds to ventricular dilation. Previously studies have shown that hydrocephalus is associated with reduction in cerebral blood flow, impairment in myelin production, and progressive loss of periventricular axons [27], and result in progressive motor dysfunction. The impairment in cognition by hydrocephalus is due to the destruction of the fimbria/fornix connections, which is critical for cognitive functions, rather than a direct effect on the hippocampus [28]. However, wire-hanging was not substantially impaired in hydrocephalic animals than non-hydrocephalic animals. And we also have shown hydrocephalus has no effect on body weight gain after $\mathrm{SAH}$. The possible explanation is rats learn to accommodate to their disability quite well $[29,30]$ and wirehanging might not be a sufficient sensitive indicator of motor and balance. In the rotarad test, we observed a clear difference in the gait and posture, quantifiable gait performance between hydrocephalic animals than non- hydrocephalic animals.

In this study, we didn't observe beneficial effects of $\mathrm{HBO}$ on hydrocephalus in the SAH animals. There are some limitations in this study. First, the sample size was 

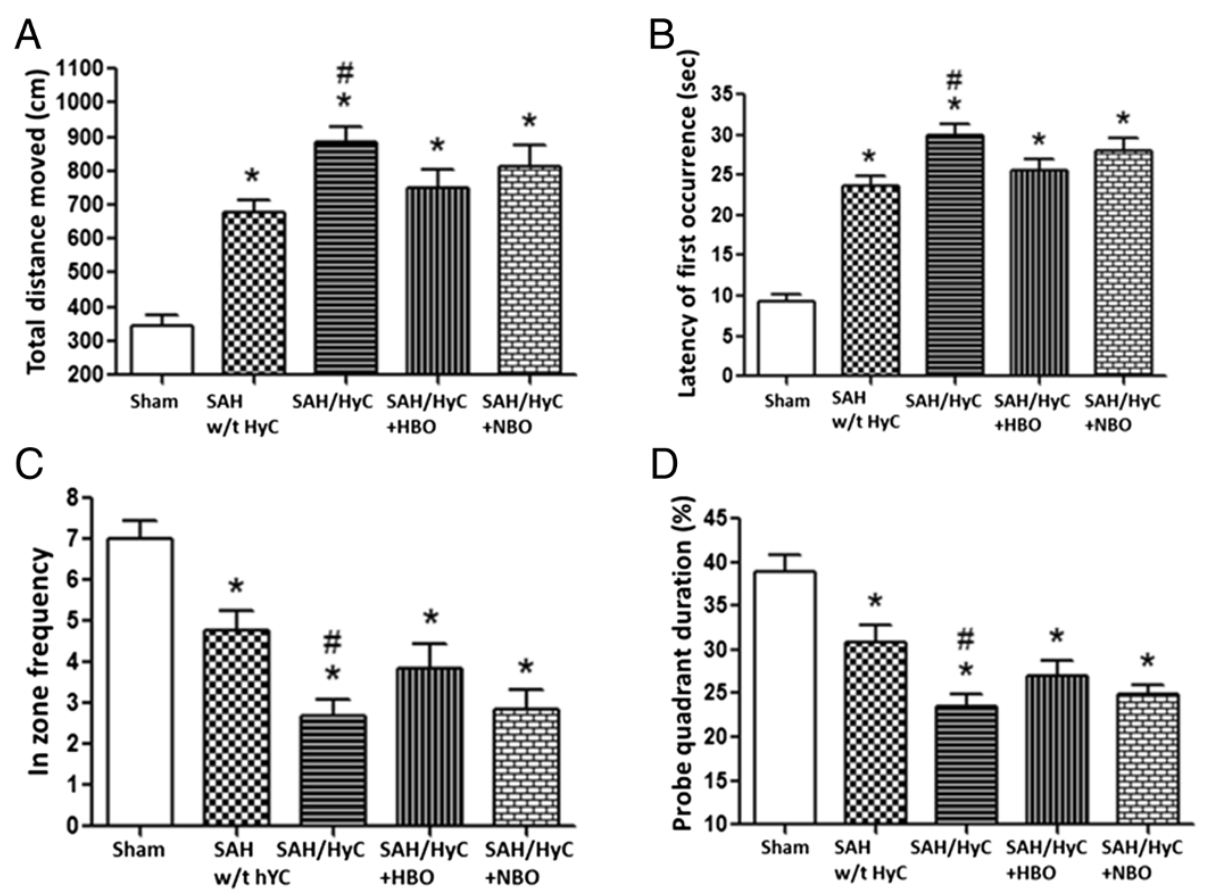

Figure 4 Multiple HBO treatment improved spatial learning and memory abilities in hydrocephalic animals. When compared with Sham group, the animals in SAH w/t HyC group had a significantly greater distance moved from the target (A), need more time to reach the platform (B), showed less frequency in target quadrants (C), spent less time in the probe quadrants (D). Hydrocephalus exacerbated the performance. Multiple HBO treatment did not showed significant improvement of memory and learning abilities when compared with $\mathrm{SAH} / \mathrm{HyC}$ group, but removed the difference between SAH w/t HyC group. Sham, $n=8$; SAH w/t HyC, $n=6 ; S A H / H y C, n=4 ; S A H / H y C+H B O, n=4 ; S A H / H y C+N B O$, $n=4 .{ }^{*} p<0.05$ compared with Sham, \#p<0.05 compared with SAH w/t HyC.

small and it might be not powerful enough to get the statistical significance. Second, we gave HBO treatment at 24 hours after $\mathrm{SAH}$, which is a delayed treatment. It has been reported that $\mathrm{HBO}$ is efficient in transient MCAO within the first 6 hours [31] and future studies on the therapeutic window of $\mathrm{HBO}$ on hydrocephalus in $\mathrm{SAH}$ animals should be investigated. Third, increasing the number of $\mathrm{HBO}$ exposures can promote the effects of $\mathrm{HBO}$ [32]. In this study we administrated HBO once for each day and multiple-exposure protocols might be more effective.

In conclusion, in the present study we proved that about $40 \%$ animals developed to hydrocephalus and showed behavioral and cognitive deficits after SAH. HBO treatment showed minor improvement in neurobehavioral deficits and cognitive functions, and did not affect ventricular volume after SAH. We provide therapeutically relevant data that HBO had no effects on the development of hydrocephalus in SAH model. The improvement in neurological functions may be resulted from the neurogenesis after multiple HBO treatment which has been proved in experimental stroke [11]. This work suggests additional studies should revisit the effective therapy strategies for hydrocephalus in clinical trials after SAH.
Competing interests

All authors declare that they have no competing interests to disclose.

\section{Authors' contributions}

QH participated in study design, data analysis and manuscript preparation; AV participated in surgery and data collection; BL participated in data collection and data analysis; JT participated in study design; JHZ participated in study design and manuscript preparation. All authors read and approved the final manuscript.

\section{Funding}

This research was conducted with funding by a grant from NIH NS043338 to J.H. Zhang.

Received: 24 March 2014 Accepted: 27 June 2014

Published: 9 July 2014

\section{References}

1. Jartti P, Karttunen A, Isokangas JM, Jartti A, Koskelainen T, Tervonen O: Chronic hydrocephalus after neurosurgical and endovascular treatment of ruptured intracranial aneurysms. Acta Radiol 2008, 49:680-686.

2. Symss NP, Oi S: Theories of cerebrospinal fluid dynamics and hydrocephalus: historical trend. J Neurosurg Pediatr 2013, 11:170-177.

3. Dorai Z, Hynan LS, Kopitnik TA, Samson D: Factors related to hydrocephalus after aneurysmal subarachnoid hemorrhage. Neurosurgery 2003, 52:763-769. discussion 769-771.

4. Massicotte EM, Del Bigio MR: Human arachnoid villi response to subarachnoid hemorrhage: possible relationship to chronic hydrocephalus. J Neurosurg 1999, 91:80-84. 
5. Sajant J, Heikkinen E, Majamaa K: Rapid induction of meningeal collagen synthesis in the cerebral cisternal and ventricular compartments after subarachnoid hemorrhage. Acta Neurochir 2001, 143:821-826.

6. Germanwala AV, Huang J, Tamargo RJ: Hydrocephalus after aneurysmal subarachnoid hemorrhage. Neurosurg Clin N Am 2010, 21:263-270.

7. Wilson HD, Wilson JR, Fuchs PN: Hyperbaric oxygen treatment decreases inflammation and mechanical hypersensitivity in an animal model of inflammatory pain. Brain Res 2006, 1098:126-128.

8. Lipsky BA, Berendt AR, Deery HG, Embil JM, Joseph WS, Karchmer AW, LeFrock JL, Lew DP, Mader JT, Norden C, Tan JS, Infectious Diseases Society of America: Diagnosis and treatment of diabetic foot infections. Plast Reconstr Surg 2006, 117:212S-238S.

9. Lee KL, Niu KC, Lin MT, Niu CS: Attenuating brain inflammation, ischemia and oxidative damage by hyperbaric oxygen in diabetic rats after heat stroke. J Formos Med Assoc 2013, 112:454-462.

10. Ostrowski RP, Zhang JH: Hyperbaric oxygen for cerebral vasospasm and brain injury following subarachnoid hemorrhage. Trans/ Stroke Res 2011 2:316-327.

11. Mu J, Ostrowski RP, Soejima Y, Rolland WB, Krafft PR, Tang J, Zhang JH: Delayed hyperbaric oxygen therapy induces cell proliferation through stabilization of camp responsive element binding protein in the rat model of mcao-induced ischemic brain injury. Neurobiol Dis 2013, 51:133-143.

12. Garcia JH, Wagner S, Liu KF, Hu XJ: Neurological deficit and extent of neuronal necrosis attributable to middle cerebral artery occlusion in rats. Statistical validation. Stroke 1995, 26:627-634

13. Zhou Y, Lekic T, Fathali N, Ostrowski RP, Martin RD, Tang J, Zhang JH: Isoflurane posttreatment reduces neonatal hypoxic-ischemic brain injury in rats by the sphingosine-1-phosphate/phosphatidylinositol-3-kinase/ akt pathway. Stroke 2010, 41:1521-1527.

14. Shabani M, Larizadeh MH, Parsania S, Asadi Shekaari M, Shahrokhi N: Profound destructive effects of adolescent exposure to vincristine accompanied with some sex differences in motor and memory performance. Can J Physiol Pharmacol 2012, 90:379-386.

15. Lekic T, Hartman R, Rojas H, Manaenko A, Chen W, Ayer R, Tang J, Zhang JH: Protective effect of melatonin upon neuropathology, striatal function, and memory ability after intracerebral hemorrhage in rats. J Neurotrauma 2010, 27:627-637.

16. Lackner P, Vahmjanin A, Hu Q, Krafft PR, Rolland W, Zhang JH: Chronic hydrocephalus after experimental subarachnoid hemorrhage. PLoS One 2013, 8:e69571.

17. Oreskovic D, Klarica M: Development of hydrocephalus and classical hypothesis of cerebrospinal fluid hydrodynamics: Facts and illusions. Prog Neurobiol 2011, 94:238-258.

18. Li T, Zhang P, Yuan B, Zhao D, Chen Y, Zhang X: Thrombin-induced tgf-beta1 pathway: a cause of communicating hydrocephalus post subarachnoid hemorrhage. Int J Mol Med 2013, 31:660-666.

19. Chen X, Duan XS, Xu LJ, Zhao JJ, She ZF, Chen WW, Zheng ZJ, Jiang GD: Interleukin-10 mediates the neuroprotection of hyperbaric oxygen therapy against traumatic brain injury in mice. Neuroscience 2014, 266:235-243.

20. Lee YS, Chio CC, Chang CP, Wang LC, Chiang PM, Niu KC, Tsai KJ: Long course hyperbaric oxygen stimulates neurogenesis and attenuates inflammation after ischemic stroke. Mediators Inflamm 2013, 2013:512978.

21. Kendall AC, Whatmore JL, Winyard PG, Smerdon GR, Eggleton P: Hyperbaric oxygen treatment reduces neutrophil-endothelial adhesion in chronic wound conditions through s-nitrosation. Wound Repair Regen 2013, 21:860-868.

22. Rinaldi B, Cuzzocrea S, Donniacuo M, Capuano A, Di Palma D, Imperatore F, Mazzon E, Di Paola R, Sodano L, Rossi F: Hyperbaric oxygen therapy reduces the toll-like receptor signaling pathway in multiple organ failures. Intensive Care Med 2011, 37:1110-1119.

23. Chang KY, Tsai PS, Huang TY, Wang TY, Yang S, Huang CJ: Ho-1 mediates the effects of hbo pretreatment against sepsis. J Surg Res 2006, 136:143-153.

24. Vlodavsky E, Palzur E, Soustiel JF: Hyperbaric oxygen therapy reduces neuroinflammation and expression of matrix metalloproteinase-9 in the rat model of traumatic brain injury. Neuropathol Appl Neurobiol 2006 $32: 40-50$
25. Calvert JW, Yin W, Patel M, Badr A, Mychaskiw G, Parent AD, Zhang JH: Hyperbaric oxygenation prevented brain injury induced by hypoxia-ischemia in a neonatal rat model. Brain Res 2002, 951:1-8.

26. Yin D, Zhou C, Kusaka I, Calvert JW, Parent AD, Nanda A, Zhang JH: Inhibition of apoptosis by hyperbaric oxygen in a rat focal cerebral ischemic model. J Cereb Blood Flow Metab 2003, 23:855-864.

27. Del Bigio MR: Pathophysiologic consequences of hydrocephalus. Neurosurg Clin N Am 2001, 12:639-649.

28. Del Bigio MR, Wilson MJ, Enno T: Chronic hydrocephalus in rats and humans: white matter loss and behavior changes. Ann Neurol 2003, 53:237-246.

29. Farr TD, Wegener S: Use of magnetic resonance imaging to predict outcome after stroke: a review of experimental and clinical evidence. J Cereb Blood Flow Metab 2010, 30:703-717.

30. Will B, Galani R, Kelche C, Rosenzweig MR: Recovery from brain injury in animals: relative efficacy of environmental enrichment, physical exercise or formal training (1990-2002). Prog Neurobiol 2004, 72:167-182.

31. Lou M, Eschenfelder CC, Herdegen T, Brecht S, Deuschl G: Therapeutic window for use of hyperbaric oxygenation in focal transient ischemia in rats. Stroke 2004, 35:578-583.

32. Singhal $A B$ : Oxygen therapy in stroke: past, present, and future. Int J Stroke 2006, 1:191-200.

doi:10.1186/2045-9912-4-12

Cite this article as: Hu et al: Hyperbaric oxygen therapy fails to reduce hydrocephalus formation following subarachnoid hemorrhage in rats. Medical Gas Research 2014 4:12.

\section{Submit your next manuscript to BioMed Central and take full advantage of:}

- Convenient online submission

- Thorough peer review

- No space constraints or color figure charges

- Immediate publication on acceptance

- Inclusion in PubMed, CAS, Scopus and Google Scholar

- Research which is freely available for redistribution

Submit your manuscript at www.biomedcentral.com/submit
C) Biomed Central 